Zdzisław Pawlak

\title{
Distributed information systems
}

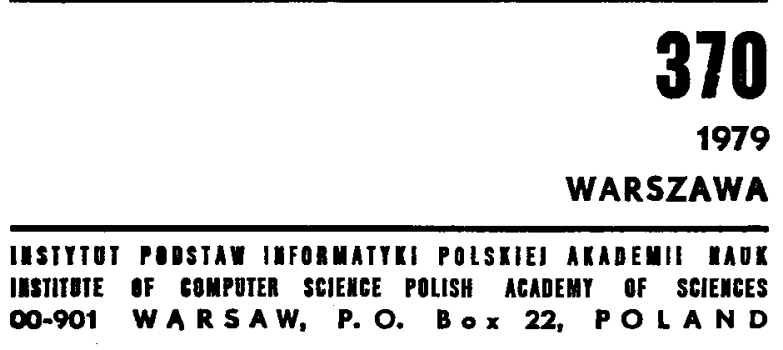


Zdzisław Pawlak

DISTRIBUTED INFORMATION SYSTEMS

370

Warsaw 1979 


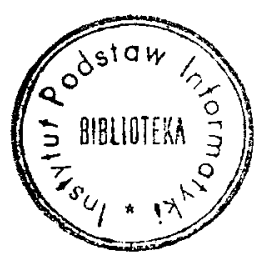

$R$ a d a $R$ e d $3 k$ c y $f$ a

A. Bikle (przewodniczący), S. Bylka, J. Lipsk1 (sekretarz), i. Jukaszenicz, R. Marczyńaki, A. Lazurkiewicz, T. Nonicki, 2. Pawlak, D. Sikorski, Z. Szoda, M. Farmus (zaatępca przemodniczęcęo)

Prace zgtosiz Andrzej Blikle

Gijirg adoreas: prof. dr hab. Zàzistan Fanlak Institute of Coraputer Sctence PAS P.O. Eox 22

00-901 Varben

Sygn. $61426 / 370 \mathrm{nr}$ inw. $2966 \mathrm{l}$

$$
\text { Frinted a a manucript }
$$

Na prawacb rekopia $u$

Nakład 700 egz. Ark. fyd. 1,20; ark. druk. 1,75. Eapier offset. $\mathrm{kl}$. III, $70 \mathrm{~g}, 70 \times 100$. Oddano do druku w Iipcu 1979 r. W. D. H. Zar. nr 449/0/79

\section{Abstract .Cодеpranue . Streszczenie}

In this paper the following problem is considered: Given $n$ "Iocal" information systems $s_{1}, \ldots, s_{n}$, with corresponding query languages $I_{1}, \ldots, I_{n}$. Find an answer to the query in a "central" language $L=U L_{1}$ as a function of local answerg (i.e. answers of local systems). Store organization related to this problem is albo brielly discussed.

\section{Распределенные ндіормационые системн}

В работе рассматривается проблема, каким обрззом найя огтот в распределенных пнорормапнонных системах на основе получеми qастичных ответов из локальных систем. Кратко обсуппесте ортанизапия памяти, связана с этой проблемой.

\section{Rozproszone systemy informacyjno}

T pracy rozwaza alę problem, w jaki sposób znaleźć odpowiedź - rozproszonych aystemach informacyjnych na podstawle uzyakiwania odpowiedz1 częśclowych z systemów lokalnych. Dyskutowena jeat krótko równiez organizacja pamięci zwiqzana z typi problemem. 
We shall deal in this note with the following problea:

We are given $n$ local inforcation gystems $s_{1}, s_{2}, \ldots, s_{n}$. With oach syotem $S_{i}$ there is associated an information language $I_{S_{1}}$ (or shortly $I_{1}$ ). The language $I_{i}$ is uged to ask queries about some informations contained in the system $S_{i}$. If $t$ is a query in $L_{i}$, then the answer to this query will be denoted by $\sigma s_{i}(t)$ (or simply by $\left.\sigma_{i}(t)\right)$.

Tie may combine systems $s_{1}, s_{2}, \ldots, s_{n}$ in one system, which we shall denote by $S$, and call global information gystem. A global information system $S$ consigting of local systems $S_{i}$ may be assumed to be for example a net of the systems $S_{i}$. 7ith the global system $S$ we may associate? global language $I_{S}$ (or in short $L$ ). The global language I may be viewed as a certain combination of the languges $I_{i}$ (local language日).

-The question arises whether we can obtain an answer to the query in global language (global query), by means of corlm bining the local answers to local queries, that is whother the sngwer to the query $t$ may be presented in the forn.

$\sigma_{S}(t)=\varphi\left(\sigma_{S_{1}}\left(t_{1}\right), \ldots, \sigma_{S_{s}}\left(t_{2}\right), \ldots, \sigma_{S_{n}}\left(t_{n}\right)\right)$,

where $t_{i}$ is a "projection" of the query $t$ on the language $L_{i}$.

1) The peper has been presented on the serinar led by prof. H. J. Schneider in Technische Unfversitut Eerlin on Werch $6,1979$. 
That is to mean that in order to answer a global query $t$ we replace the query $t$ by some queries $t_{1}, t_{2}, \ldots, t_{n}$, referring to corresponding local systems, and afterwords we form the global answer from the local answers obtained in this way. For example, let us assume that we have information systems owned by insurance company, medical'care, service bank office, police etc. In each such a system we may answer specific queries related to the needs of the owner of the system, like "liat ali persong with the seving account greater than $10000 \mathrm{gn}$ or "list all persone which had a car accident in 1977" e'tc. Each such a query is related to specific information system, in which the relevant information are stored. However, it may happen that some system user may be also interested in obtaining informations from geveral various bystems, for example he may agk"whether there are persons who have caused a car accident while being treated with some drugs". This kind of queries cannot be answered by searching files in only one information system. This information is distributed at least in two gystems: medical care gystem and insurance company system (or police system). Thus in order to get an answer to such a query we have to search for some information in more than one system. Of coursa the query language of each local system does addmit such general queries as given in the recent example. So we must also have an appropriate query language tailored properly to admit general queries.

The problem stated above is connected with anotiner one whether every local user is allowed to ask general queries or, in other words, whether any local user has access to informations stored in another system. If not and this is widely used practice the question is how "restrict acceso to protected informations against an unauthorised uger. This problem will not be considered here, however we shall nake some remarks concerning this subject.

Similar problem was also investigatyed by Marek [1], however there are some essential differences in our approach. We are mainly aiming at a logical analysis of distributed systems and their languages, whereas harek's paper was devoted mainly to the problem of organizing the data base machine".

We shall use standard mathematical notation throughout the paper.

We shall use the basic notions concerning information system after $[2]$.

\section{Infornation system}

In this paper we shall uge the notion of an information gystem introduced in [2]. Although the relevant definition is rather simple and does not cover some interesting cases of information system, however it expresses the most fundamental features of information syatems and allows to investigate many basic properties of such systems.

By an information system ie shall mean a 4-tuple

$$
S=\langle x, A, V, \zeta\rangle,
$$

\section{where}

X - is a finite set of oqjects,

A - is a finite set of attributes,

$V=\bigcup_{a \in A} V_{a}$ is the domain of attribute a for the get of values at attribute al. 
$-8-$

We assume that each attribute has at least two values. $\zeta^{-}$is an information function from $I \times A$ into $V$, such that $\rho(x, a) \in \nabla_{a}$ for every $x \in I$ and every $a \in A$;

$$
v_{a}=\left\{v \in \nabla: \bigvee_{x} \zeta^{(x, a)=v}\right\}
$$

Any pair $(a, v), a \in A, v \in V_{a}$ will be called a descriptor of attribute $a$.

For any $x 6 X$, by $\zeta_{x}$ we denote a function from $A$ into $v$ auch that $\rho_{x^{(a)}}=\rho^{(x, a)}$. Thia function will be called the information about $x$ in $s$. (Information about $x$ in. $S$ may be also called the document about $x$. in $S$ or data about $x$ in $S$ ).

For a given information syatem we introduce two binary relations, $: \widetilde{a}, \widetilde{s}$ defined as follows:

$$
\begin{array}{ll}
x \widetilde{a}^{y} \text { iff } & \rho^{(x, a)}=\rho^{(y, a)} \\
x \widetilde{s} y \text { iff } & \zeta_{x}=g_{y} .
\end{array}
$$

One can aimply check that both $\widetilde{a}$ and $\widetilde{s}$ are equivalence relations on $X$ and

$$
\text { relations on } \widetilde{s}=\bigcap_{a \in A} \widetilde{a},
$$

where $\bigcap$ is a product of partitions (equivalence relations) defined in the usual way.

The relation $\widetilde{S}$ will be also denoted by $\widetilde{A}$. SimilarIy, for ang $A^{\circ} \subset A^{\prime} \widetilde{A^{\prime}}$ is to mean $\bigcap_{a_{i} \in A^{*}} \widetilde{a_{i}}$.

The family of equivalence classeg $x / \widetilde{S}$ will be denoted by $E_{S}$ (when $X$ is fixed) and the equivalence classes will

$$
-9-
$$

be called elementary sets in $S$ for, when $S$ is pixed, -

elementary sets).

Similarly, for any $a \in A, E_{A}$ is to mean the family of equivalence clasees of the relation $\tilde{a}$.

Empty set $\phi$ and every get being an union of gome elementery. gets in $S$ will be called degcribable set in $S$. The class of an describable sets in $S$ will be denoted by $D_{3}$.

\section{Attributes}

Let $a, b \in A$ be two attributes in an information system $S=\langle X, A, \nabla, B\rangle$.

Aitribute $b$ ls said to be dependent on a ( $a \Rightarrow b)$ iff $\widetilde{a} \subset \widetilde{b}$. Attributes $a, b$ are said to be equivalent $(a \approx b)$ iff $\widetilde{a}=\widetilde{b} \cdot$

Attributea $a, b$ are called independent iff neither $\widetilde{a} \widetilde{b}$ nor $\widetilde{a} \supset \widetilde{b}$ holds.

Similarly we introduce the relations $B \Rightarrow a, 3 \Rightarrow \bar{B}$, $B \Rightarrow C$, where $B, C$ are subsets of $A$.

Attribute a is said to be depenuent on the set of attributes $B \subset A, B=\left\{b_{1}, b_{2}, \ldots, b_{n}\right\}$ ift $\widetilde{B} \subset \widetilde{a}$, and $\Rightarrow B$ iff $\sim \subset \subset \widetilde{B}$. In general we may write $B \Rightarrow C$ iff $\widetilde{B} \subset \widetilde{C}$.

Tne meaning of the "depending" relation $B \Rightarrow C$ is obvious. It simply means that values of the left hand side attributes determine values of the right hand side attributes. That is to say, if $B \Rightarrow C$, then there exists exactly one function

$$
\mathbf{f}: \prod_{b \in B} \nabla_{b} \rightarrow \prod_{c \in C} \nabla_{c}
$$


such that

$$
\left(g^{(x, c))_{c \in C}}=f\left(\left(\rho^{\left.(x, b)_{b \in B}\right)}\right.\right.\right.
$$

In other words there exigts exactily one get of functions

$\left(f_{c}\right)_{c \in C}$, such that

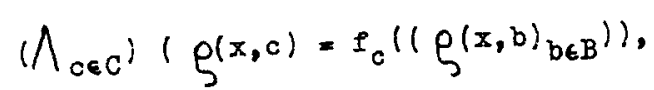

and

$$
\rho^{(x, c)}=f_{c}(\rho(x, b) b \in B)
$$

iff

$$
\begin{aligned}
& x_{c}, \rho(x ; c) \geq x_{b,} \rho^{\left(x, b_{1}\right) \cap x_{b_{2}}, \rho^{\left(x, b_{2}\right)} \cap \cdots}
\end{aligned}
$$

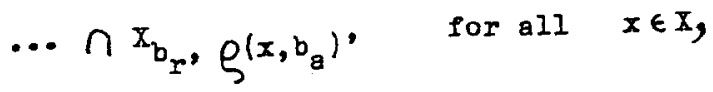

where

$$
x_{c, x}=\left\{x \in X: \rho_{x}(c)=v\right\} .
$$

Besides the functions $f_{c}, e \in C$ we shall also use functions $f_{\mathrm{C}}^{\#}$, with the following domains and codomaing

$$
\text { f: } \prod_{b \in B} E_{b} \longrightarrow E_{c}
$$

defined as follows:

$$
x_{c}, \rho^{(x, c)=f_{c}^{3 \pi}\left(\left(x_{b}, \rho(x, b)\right)_{b \in B}\right)}
$$

$$
g^{(x, c)}=f_{c}\left(g^{\left.(x, b)_{b \in B}\right)}\right.
$$

for all $x \in \mathbb{X}$.

\section{REDUCED SYSTEMS}

As we have stated in the previous section, same attributes in the information sygtem may be superfluous, in this sence that their values can be "cerived" from the values of other stributeg in the system. A natural question, arises whether we can remove all dependent attributes from the system and what properties has the system obtained in this way. Such a system will be called a reduced information system. In this paper we shall only give a definition of a reduced system; some basic properties of reduced systems will be published alsewhere.

$$
\text { Let } S=\langle X, A, \nabla, \rho\rangle \text { be an information system. }
$$

$A$ subset $B \subseteq A$ is called independent in $S$ iff, for every $B^{0} \mathfrak{F} B \quad \widetilde{B} \neq \widetilde{B}$.

If $B \subseteq A$ is independent in $S$, then for every $B^{\circ} C B$ and every $a \in B-B^{\circ} B \nRightarrow a$.

$A$ subset $B \subseteq A$ is said to be dependent in $S$ iff there exists a subset $B^{\circ} \mathcal{F}$ such that $\widetilde{B^{\prime}}=\widetilde{B}$.

If $B$ is dependent in $S$ then there exists $B^{\circ} C B$ and

a $B-B^{\prime}$ such that $B \Rightarrow a$.

The get $A$ is asid to be derivable from $A^{\circ}$ iff $A^{\circ} C A$

and $\widetilde{A}=\widetilde{A^{\prime}}$.

If $A^{\circ} \subset A$ and $A$ is derivable from $A^{*}$ in $S$ then

$A^{\circ} \Rightarrow A-A^{\circ}$. 
A set $A^{\circ} C A$ will be called a reduct of $A$, iff $\widetilde{A}=\widetilde{A^{\prime}}$ and theire does not exiat a proper aubset $B$ of $A^{\prime}$ such that $\widetilde{B}=\widetilde{A^{\prime}}$.

The corresponding system $S=\left\langle X, A^{\prime}, \nabla, \rho^{\prime}\right\rangle$ will be called a reduced system $\left(\rho^{\prime}\right.$ is the restriction at the function $S$ to the aet $\left.X \times A^{\circ}\right)$.

Naturalzy, a given system may have more than one reduct. In the sequel we shall consider only reduced information system, unless atated to the contrary.

4.: SUBSYSTEMS AND APPROXIMATIONS

$$
\text { Let } s=\langle x, A, v, \rho\rangle \text { and } s^{*}=\left\langle x^{*}, A^{*}, v^{*}, \rho^{\prime}\right\rangle
$$

be two information systems.

We say that $S^{\circ}$ is a subsyatem of $S\left(S^{*}<S\right)$ if $X^{*} \subset X, A^{*} \subset A, \quad V^{\circ} \subset \nabla$, and

$$
\rho^{\prime}=\zeta / X^{\prime} \times A^{\circ}
$$

If $S^{\circ}<S$ and $X^{*}=X$ we shall say that $S^{\circ}$ is an attribute regtricted subsyatem of $S$ (in short a.r. system). If $S^{\circ}<S$ and $A^{\circ}=A, \nabla^{*}=V$, and

$$
\rho^{\prime}=\zeta / x^{\prime} \times A^{-}
$$

then $S^{\circ}$ will be refered to as an object restricted gubsystem

of (in short o.r. Bystem).

If $s^{\prime}<s$ and $s^{\prime}$ is a.r. oystem then $\widetilde{s}^{\prime} \supset \tilde{s}^{\cdot}$

If $S^{*}<S$ and $S^{\prime}$ is o.r. system then

$$
\tilde{s}^{\prime}=\tilde{s} \bigcap\left(x^{*}\right)^{2}
$$

or, in other words, for any $a^{\circ} \in E_{g}$, there exists $e \in E_{g}$ such that $e^{*}=e n\left(X^{*}\right)^{2}$.

$$
\text { Let } S=\langle x, A, V, \rho\rangle \text { and } S^{\circ}=\left\langle x, A^{\circ}, \nabla^{\prime}, \rho^{\prime}\right\rangle
$$

be two information syatems and let $S^{\circ}$ be a.r. subsystem of $S$. Let $Y \in D_{S}$ and $Z \in D_{S} \cdot$. Thus $Y$ and $Z$ are sums of some elementary sets in $S$ and $S^{*}$, respectively.

$Z$ will be called an upper approximation of $Y$ in $S^{\prime}$ if $Y \subset Z$.

If $Z$ is an approximation of $Y$ in $S^{\prime}$ and there does not exist a proper subset $Z^{\prime} \subset Z$ such that $Y \subset Z^{*}, Z^{\prime} \in D_{S^{\prime}}$, then $z$ will be called the best upper approximation (b.u.a.) of $Y$ in $S^{\prime}$.

If $z$ is the b.u. $a$. of $Y$ in $S^{\prime}$ we shall write

$$
\mathrm{Z}=|\mathrm{Y}|_{\mathrm{S}^{\prime}}^{\mathrm{*}}
$$

It is easy to prove the following property:

$$
\text { Let } S=\langle X, A, \nabla, g\rangle \text { and } S^{\prime}=\left\langle X, A^{\prime}, V^{\prime}, g^{\prime}\right\rangle
$$

be two information syatems, and let $S^{\circ}$ be an attribute restricted subsystem of $S$. Then for every $Y \in D_{S}$ there exists exactly one b.u.a. $Z$ of $Y$ in $S^{\circ}$, namely,

$$
Z=|Y|_{S}^{\bar{x}}=\bigcup_{i=1}^{k} f^{X}\left(Y_{i}\right) \text {, }
$$

where

$$
Y=\bigcup_{i=1}^{k} Y_{i}, \quad Y_{i} \in E_{S},
$$

and

$$
f^{x}: E_{S} \rightarrow E_{S^{-}}
$$


is such that for any $e \in E_{S^{\prime}}, e^{0} \in E_{S^{\prime}} f^{\pi}(e)=e^{\prime}$, iff $e \subset e^{*}$.

The proof is by induction on the number of elementary sets in $Y$.

We shall also need the notion of the best lower approximation (b.1.a.) of $Y$ by $z$ in $S^{\prime}$.

$$
\text { Let } S=\langle X, A, \nabla, \rho\rangle \text { and } S^{\prime}=\left\langle x^{\prime}, A, \nabla, g^{\prime}\right\rangle
$$

be information systems, and let $S^{\prime}$ be object reatricted

subsystem of $S$. Let $Y \in D_{S}$ and $Z \in D_{S} \cdot Z$ will be called the best lower approximation of $Y$ in $S^{\prime}$ iff $Y \supset Z$ and there dóes not exist $Z^{\circ} \supset Z$ such that $Z^{\circ} \in D_{S}$ and $Z^{\circ} \subset Y$. The b.1.a. $Z$ of $Y$ in $S^{\circ}$ will be denoted as

$$
Z=|Y|_{\text {ss }} \mathrm{S}^{\prime \bullet}
$$

The following property is valid:

$$
\text { Let } S=\langle\mathrm{X}, \mathrm{A}, \mathrm{V}, \mathrm{g}\rangle \text { and } \mathrm{S}=\left\langle\mathrm{X} ; \mathrm{A}, \mathrm{V}, \mathrm{g}^{\prime}\right\rangle
$$

be two information systems, and let $S^{\prime}$ be object restricted

subsystem of $S$. Then to every $Y \in D_{S}$ there exist exactly

one br.a. $Z$ of $Y$ in $S^{\circ}$, and

$$
z=|Y|_{* S^{\prime}}=\bigcup g^{K}\left(Y_{i}\right) \text {, }
$$

where

$$
Y=\bigcup_{i=1}^{h} Y_{i}, \quad Y_{i} \in E_{S}
$$

and

$$
E^{\pi}: E_{S} \rightarrow E_{S^{\prime}} \cup\{\phi\}
$$

is defined as follows:

$$
g^{* x}(e)=e n\left(x^{\prime}\right)^{2}
$$

The proof is also by induction on the number of elementary sets in $Y$.

\section{JOIN OF INFORAATION SYSTEMS}

If $\mathrm{s}_{1}, \mathrm{~S}_{2}, \ldots, \mathrm{s}_{\mathrm{k}}$ are information systems, we can define a "common" information syatem $s$ guch that $s_{1}, s_{2}, \ldots, s_{h}$ ara subsystems of $s$. The syetem $s$ will be called join of $S_{1}$ and will be denoted as $s=\bigcup_{i=1}^{k} s_{i}, s_{i}=\left\langle x_{i}, A_{i}, v_{i}, \rho_{i}\right\rangle$.

the join $S$ of $S_{i}$ is defined in the following way:

$$
\mathrm{s}=\langle\mathrm{x}, \mathrm{A}, \mathrm{v}, \rho\rangle
$$

where

$$
\begin{aligned}
& X=\bigcup_{i=1}^{k} x_{i} \\
& A=\bigcup_{i=1}^{k} A_{i} \\
& V=\bigcup_{i=1}^{k} V_{i}
\end{aligned}
$$

$$
\rho^{\prime} x_{i} \times A_{i}=\varphi_{i} \quad i=1, \ldots, k
$$

or $\rho_{x}=\bigcup_{i=1}^{k} \rho_{i x}$, for all $x \in X$.

The join $s$ of $s_{i}$ is well defined if the following two conditions are satisfied: 
$1^{0} \cdot\left(\bigwedge_{i, j}\right)\left(x_{i} \cap x_{j} \neq \phi\right.$ and $\quad A_{i} \cap A_{j} \neq \phi \rightarrow$

$$
\rho_{i} /\left[\left(x_{i} \cap x_{j}\right) \times\left(A_{i} \cap x_{j}\right)\right]=
$$

$$
\rho_{j} /\left[\left(x_{i} \cap x_{j}\right) \times\left(A_{i} \cap A_{j}\right)\right],
$$

and

$2^{\circ}$. $\left(\bigwedge_{I \in X}\right)\left(\rho_{x}=\bigcup_{i=1}^{k} \rho_{i x} \quad\right.$ is defined for an $a \in A$.

The first condition is obvious and the second need some explanations.

Let $S_{1}$ be a gystem with only one attribute, say colour and $S_{2}$ a gyatem also with one attribute for example length and assume that $x_{1} \cap x_{2}=\varnothing$. The condition $2^{\circ}$ eays that we are not allowed to define join $s$ of $s_{1}$ and $s_{2}$ because we do not have any information about lengths of objects in $s_{1}$ and about colours of objects in $S_{2}$. Thus wo are unable to define for all $x \geq$ the information $\xi_{x}$ about colour and length of $x$. In other words, we are not able to define the function $\zeta_{x}$ for the join $s=s_{1} \cup s_{2}$

This seems to hove neturel justification in reallife gyatems. If we have two information syatema, ay first concerning insurance and the second medical care with different sets of population (for example one in Iondon and the second in Warsaw), then combining these two gystems into one joint gystem is justified only in the case when we have insurance data in medical gystem and conversely. Othermige we are unable to define for all $x \in X$ the information $\zeta_{x}$ about insurance and medical care and, conequently, according to our definition the join of these two gystems in not an informstion gyatem.
Evidently if $s=\bigcup_{1=1}^{k} s_{1}$, then

$$
\widetilde{s}=\bigcap_{i=1}^{k} \widetilde{s}_{1}
$$

Moreover, every $S_{1}$ is a subagstem of $S$.

We shell distinguigh two kinde of joins $s$.

In the first case we assume that every subsystem $S_{i}$ of the system $S$ is attribute restricted and such a joint system will be called an attribute joined sygtem (or an attribute join of $\left.s_{1}, \ldots, s_{n}\right)$.

The second case referg to the syatem $S$ in which every

$S_{i}$ is object restricted subsyatem of $S$. This kind of aystem we will refer to as an object joined gystem (or an object join

of $\left.s_{q}, \ldots, s_{n}\right)$

Thue in an attribute joined $s=\bigcup_{i=1}^{k} s_{1}$

$$
s=\langle\mathbf{x}, A, \nabla, \rho\rangle
$$

we have the subaystems

$$
s_{i}=\left\langle x, A_{1}, v_{i}, \rho_{i}\right\rangle
$$

and in the object joined aystem $S=\bigcup_{i=1}^{K} S_{i}$

$$
s=\langle\mathbf{x}, \Lambda, \nabla, \rho\rangle
$$

ag aubgyatems we have

$$
s_{i}=\left\langle I_{i}, \Lambda, \nabla, \rho_{i}\right\rangle .
$$


6. QUERY IAATGUAGE OF INPORMATION SYSTEM

With every information oyatem $S$ we associate a query language $I_{S}$ which will be used for alking queries about informations contained in the system $S$. For the sake of eimplicity wo conaider very bimple language, which is apart of a query language defined in

The query languge considered in this paper, will congiat only of terma, which are logical combinationa of descriptors. That is to say a term (a query) in the considered language iptor, or any expression built up from descriptore by means of logical connectives mor", "and", "not". of $I_{S}$ consigts of the following aymbols:

$1^{0} 0,1$ - constans

-

$3^{\circ} U, n, \sim$ - ermbole for logical connectives norn,

"and", nnot" respectirely

$4^{\circ}$ (.) - parentheses.

Terma. (queries) in the language $I_{S}$ are defined in the folloning manner

$$
\begin{aligned}
& 1^{0} 0,1 \text { are terms } \\
& 2^{0} \text { ang pair }(a, v), a \in \Lambda, r \in \text { is a term } \\
& 3^{0} \text { If } t, t^{\circ} \text { are term, so are } \sim(t),\left(t u t^{\circ}\right),\left(t t^{\circ}\right) \text {. }
\end{aligned}
$$

The meaning of a term $t$ (an anower to the quers $t$ ) is

a function

$$
\partial_{s}: \sigma_{s} \rightarrow p(x),
$$

$\sigma_{S}$ - is the set of all tersos in the language $I_{S}$.

The meaning is defined in the following way:

$$
\begin{aligned}
& 1^{0} b_{S}(0)=\phi ., \sigma_{S}(1)=x \text {, } \\
& 2^{0} \quad b_{S}(\theta, r)=\left\{x \in \mathbb{R}: \rho_{x^{(0)}}=v\right\} \text {, } \\
& 3^{0} b_{S}(r t)=x-b_{S}(t) \\
& b_{S}(t \cup t)=\sigma_{S}(t) \cup b_{S}\left(t^{\prime \prime}\right) \\
& \sigma_{S}\left(t \cap t^{\prime}\right)=\delta_{S}(t) \cap b_{S}\left(t^{\prime}\right) \text {. }
\end{aligned}
$$

term will be called an elementary term if it is of the form

$$
\begin{aligned}
& t=\left(a_{1}, \nabla_{i_{1}}\right) \cap\left(a_{2}, \nabla_{i_{2}}\right) \cap \ldots \cap\left(a_{n}, \nabla_{i_{n}}\right), \\
& \Delta=\left\{a_{1}, a_{2}, \ldots, a_{n}\right\}, \quad \nabla_{i_{j}} \in \nabla_{a_{j}} .
\end{aligned}
$$

If $T_{E}$ is the get of all elementary terms in $I_{S}$, then

$$
\begin{aligned}
& 1^{\circ} \quad\left(\Lambda_{t, t^{*}} \in \sigma_{E}\right)\left(\sigma_{S}(t) \cap \sigma_{S}\left(t^{*}\right)=\phi\right) \\
& 2^{0} \quad \bigcup_{t \in \tau_{E}}{ }_{s}(t)=x \text {. }
\end{aligned}
$$

Thus the set of all elementery terms generates a partition of the set $X$ (i.e. defines an equivalence relation on $X$ ).

It is easy to aee that this equivalence relation coincides vith relation $\widetilde{S}$. 
Namely, if $6 \mathrm{~S}^{(t)}$ is not empty, then ${ }_{\mathrm{S}}(t)$ is juat an equivalence class of the relation $\tilde{s}$. Thus elementary terms may be considered as unique names with nonempty valueg (in the language $I_{S}$ ) of elementary sets, (i.e. if $e, e^{\prime}$ are two different elementary sets in $S$ then the corresponding elementary terms are different twol.

term t in in normal form if it is a gum of some elementary terms, i.e.

$$
t=t_{1} \cup t_{2} \cup \ldots \cup t_{1}
$$

where $t_{i}$ - are elementary terms.

The following is true:

For every term $t$ in $I_{S}$ there exiats $t^{*} \epsilon \quad I_{S}$ such that $t^{*}$ is in normal form and

$$
\sigma_{S}(t)=\sigma_{S}\left(t^{\circ}\right)
$$

The subset $I \subset Z$ is called deacribable in $I_{S}$ iff there oxiate a term $t$ in $I_{S}$ such that

$$
\sigma_{S}(t)=I
$$

From the normal form property we can deduce that all nonempty describable sets in $I_{S}$ are unions of some elementary sets in $S$. That means the set of all describable aets in $I_{S}$ is identical ith the set $D_{S}(X)$ introduced in the first paregraph of this peper.

If $s^{*}<s$, then we ghall aag that the language $I_{S^{*}}$ is a gublangusge of $I_{S^{*}}$
If $S^{\circ}$ is ettribute reatricted subsyatem of $S$, then $I_{S}$. will be called gyntactically restricted sublanguage (s.r.s.) of $I_{S}$. If $I_{S}$, is a s.r.s. of $I_{S}$, then for every term $t$ in $I_{S}$, there exists a term $t^{\circ}$ in $I_{S}$ such that

$$
\sigma_{S}\left(t^{*}\right)=\left|\sigma_{S}(t)\right|_{S^{*}}^{*}
$$

If $S^{*}$ is object reatricted subsygtem of $S$; then both languages $I_{S}$ and $I_{S}$ are identical, but the meanings of terms $t$ in these languages are different. (Because both gyatemo" have different sets of objecta).

IramelJ, for ans terw $t$ in $I_{S}$ for in $I_{S}$ )

$$
\sigma_{S}(t)=\left|b_{S}(t)\right|_{*} s^{\prime}
$$

If $S$ is an attribute join of gyateme $S_{i}$, then each sublanguage $\mathrm{I}_{\mathrm{S}_{1}}$ is naturally a syntactical restriction of the language $\mathrm{I}_{\mathrm{S}}$.

The language $\mathrm{I}_{\mathrm{S}}$ corresponding to the join $\mathrm{S}$ of systems $S_{i}$ will be called the join of languages $I_{S_{1}}$ and denoted by

$$
I_{S}=\bigcup_{i=1}^{k} i_{S_{i}}
$$

The join of languagea $\mathrm{L}_{\mathrm{S}_{i}}$ is outained simply by admitting in the definition of the languages $I_{S}$ an attributes and values from all aublangragea $\mathrm{I}_{\mathrm{S}_{i}}$.

In an objeot joined system $S$ all sublanguages ${ }^{I} S_{i}$ are the same and the language $\mathrm{I}_{\mathrm{S}}=\mathrm{I}_{\mathrm{S}_{j}}$. 
$-22-$

In this case the syntax of esch language $I_{S_{i}} i_{\text {s exactly the }}$ same, but the mesning of two identical terms belonging to different languages is different.

7. DISTRTBUTED INFORMATION SYSTEMS

Now we shall return to our original problem stated at the beginning of this paper.

Assume we are given some information system $s_{1}, S_{2}, \ldots, s_{k}$, together with the languages $\mathrm{I}_{\mathrm{S}_{1}}, \mathrm{I}_{\mathrm{S}_{2}}, \ldots, \mathrm{I}_{\mathrm{S}_{\mathrm{n}}}$ agsociated with them.

mey think of this systems as separated units, not connected together, and used independently.

We can agsume however that that there is a kind of link between those gyatems so that they form one distributed information aystem, which may be viewed as a join of individual sygtems $S_{i}$. The language $I_{S}$ of the join aystem $S$ may be algo considered ar a join of all sublanguager. $\mathrm{I}_{\mathrm{S}_{i}}$.

we may come back now to tho original queation: wo to query in the language $I_{S}$ retrieving informationg in subsyotems $S_{i}$.

all consider in this paper two basic types of information systems.

5 will be called a language distributed system if

$$
s=\langle x, A, \nabla, \rho\rangle,
$$

and

$$
s=\bigcup_{i=1}^{k} s_{i}
$$

where
$-23-$

$$
\rho_{i}=\rho / \times \times A_{i}
$$

and

$$
A_{i} \cap A_{j} \neq \phi^{\cdot} \rightarrow \rho_{i} / X \times\left(A_{i} \cap A_{j}\right)=\zeta_{j} / X \times\left(A_{i} \cap X_{j}\right)
$$

$\mathrm{S}$ will be called data distributed gystem if

$$
S=\langle x, A, Y, g\rangle
$$

and

$$
\begin{gathered}
s=\bigcup_{i=1}^{k} s_{i} \\
s_{i}=\left\langle x_{i}, A, \nabla, \rho_{i}\right\rangle, \\
\rho_{i}=\rho^{/ x_{i} \times A}
\end{gathered}
$$

and

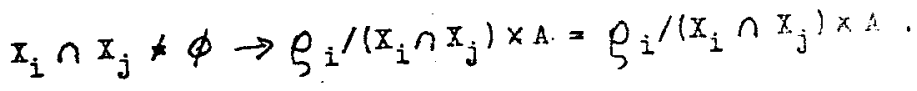

8. IANGUAGE OF DISTRIBUTED SYSTEM

Let $S=\langle X, A, \nabla, g\rangle$ be a language distribibci эystem,

i.e.

$$
S=\bigcup_{i=1}^{k} s_{i}
$$

and

$$
s_{i}=\left\langle\pi, A_{i}, \nabla_{i}, \rho_{i}\right\rangle
$$


If $t \in \mathbb{B}_{S}$ (i.e. $t$ is an elementary term in $L_{S}$ ), then by $t / A_{i}$ we shall mean the elementary term $t^{*} \in I_{S}$ obtained from $t$ after removing all descriptors which do not belong to $I_{S_{i}}$ (if after this removal no descriptors remain, then we-assume that $t / A_{i}=T$ ).

Then the value of any term $t$ in $I_{S}$ can be represent as follows

$$
\sigma_{S}(t)=\bigcup_{j=1}^{n} \bigcap_{i=1}^{k} \delta_{S_{i}}\left(t_{j} / A_{i}\right),
$$

where

$$
t_{1} \cup t_{2} \cup \ldots v t_{n} \text { ia a normal form of } t_{\text {, }}
$$

Obriously we have $\sigma_{S_{i}}\left(t_{j} / A_{i}\right)=\left|\delta_{S}\left(t_{j}\right)\right|_{S_{i}}^{*}$

This property means aimply that in order to find an answer to the query $t$ in the language $I_{S}$, we must first tranglate it into a normal form $t_{1} \cup t_{2} \cup \ldots \cup t_{n}$. Then for any $1 \leqslant 1 \leqslant k$ and any $1 \leqslant j \leqslant n$, we remove from each elementary term all degcriptora which do not belong to the language $I_{S_{1}}$, i.e. for each $1 \leqslant i \leqslant n$ we replace sll elenentary terma by terms of the form $t_{j} / A_{i}=t_{j}$ belonging to the languages $\mathrm{I}_{\mathrm{S}_{i}}$. Afterwarde we have to find the best upper approximation

$$
\left|t_{j}\right|_{i}^{*}=b_{s_{i}}\left(t_{j}^{*}\right)
$$

for each term $t_{j}$ in every subsystem $S_{i}$. Then the intersection of all approximations corresponding to a fixed elementary term $t_{j}$ constitutes the proper answer to this term.
In order to obtain the whole answer to the query $t$ we have to "add" the answerg to all elementary terms occuring in the normel form of $t$.

The situation may be depicted as shown in figure 1.

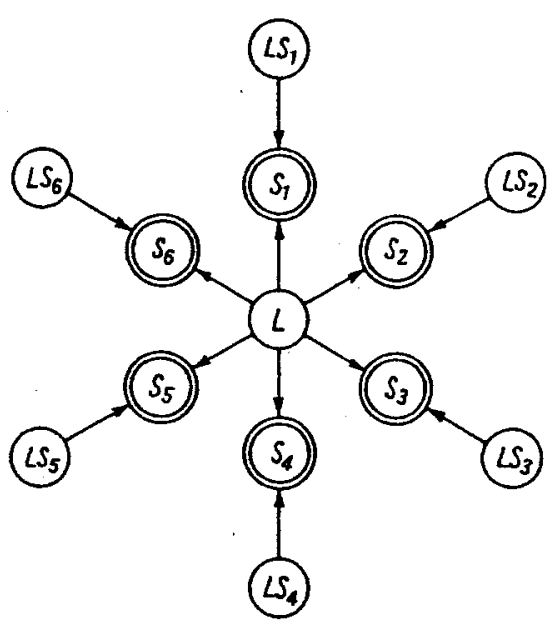

Fig. 1.

So we have in this case two kinds of users: local and global (central) ones. Local users are attached to local systens use local languages $I_{i}$, and have access only to informations in looal systems. The global uger can ask queries in the global langrage $I$ and he has accesa to the informations in all local gy日tems.

This kind of organization of distributed gystem has one serious disadvantage. In order to find the answer to a global query, one has to earch for the beat uppor approximations of elementary terms in local system and take the intersection of 
all approximations. The intersection operation is very unefficient, because it requires access to many disc memories in order to retrieve the best upper approximations of elementary terma, but only small part of thus obtained data will occur in the intersection of all approximations.

Thus another solution of this kind of systems seems to be more efficient. This solution is depicted in fig. 2 .

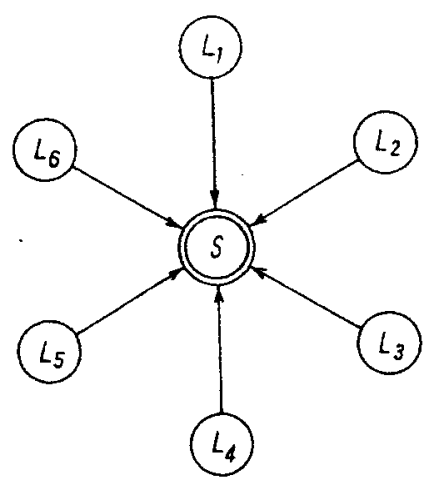

Fig. 2.

There if only one central gystems and each user uses its own language $I_{i}$ which can be any sublanguage of $I_{S^{*}}$ Because, in this case there is only one partition $\tilde{S}$ generated by tho global gystem $S$ the answer to any query in global language or any sublanguage of the global language may be obtained directly from the central memory as a sum of some elementary sets in 5 . Thus in this case there is no intersection operation, which considerably slowa down the ratrieval process.
We shall call the first type of language distributed systems - local memory sygtems. The second kind of systems congidered in this paragraph will be reierred to as central memofy gystems.

9. DATA DISTRIBUTED SYSTEMS

In this paragraph we shall deal with the case winen the subsystems $S_{i}$ of the system $S$ have the same lanellares $I_{S}$, but different seta of objects.

Let $S$ be a data distributed system i.e. let

$S=\langle X, A, V, \rho\rangle$ be a system

$$
S=\bigcup_{i=1} S_{i}
$$

where

$$
s_{i}=\left\langle x_{i}, \lambda, v, \rho_{i}\right\rangle,
$$$$
\rho_{i}=\rho^{\pi_{i} \times}
$$

and

$$
\begin{aligned}
x_{i} \cap x_{j} \neq \phi \rightarrow & \rho_{i} /\left(x_{i} \cap x_{j}\right) \times s= \\
& =\rho_{j} /\left(x_{i} \cap x_{j}\right) \times A .
\end{aligned}
$$

If $S$ is a data distributed system then for every term

$t$ in $\mathrm{I}_{\mathrm{S}}$

$$
\sigma_{S}(t)=\bigcup_{i=1}^{k} \delta_{i}(t) .
$$


obviously, we have

$$
\sigma_{S_{i}}(t)=\left|\sigma_{S}(t)\right|{ } S_{i}
$$

where the normal form of $t$ is

$$
t=t_{1} \cup t_{2} \cup \ldots, v t_{n} \cdot
$$

From this property it follows that in order to find the answer to the querry $t$ in $\mathrm{I}_{S}$ we have to find partial answers (t) in subsystems of $S$. This partial answers are sums $b_{i}(t)$ in the best lower approximations for $t$ is the sum of all partial system $S$. The answer to the querry simple to implement and is much answers. The operation is very simple to upper approximations faster then computing the intersection of upper app in the previous case. mory systems and central memory systems.

local memory systems and centas is similar and it is The implementation in all cases is simllar and it is rather simple.

\section{Acknowledgment}

Thanka are due to $d r$ B. Konikowaka for reading the manuscript and essential improvement of the paper. Schneider, Dr. P. Bollmann and D.. E. Konrad for stimulating discussion and valuable remarks and comments. 